Article

\title{
Effect of Floor Shape Optimization on Energy Consumption for U-Shaped Office Buildings in the Hot-Summer and Cold-Winter Area of China
}

\author{
Xiaoyu Ying ${ }^{1}$ and Wenzhe $\mathrm{Li}^{2, *}$ \\ 1 Department of Architecture, Zhejiang University City College, Hangzhou 310015, China; \\ yingxiaoyu@zucc.edu.cn \\ 2 Department of Architecture, Zhejiang University, Hangzhou 310058, China \\ * Correspondence: mit600_lee@163.com
}

Received: 30 January 2020; Accepted: 5 March 2020; Published: 8 March 2020

\begin{abstract}
This paper explored the effects of the side proportion of building floor shape on building energy consumption. It is based on the analysis of regression models that were developed in the present study. The simplified building models can be used to conduct a parametric study to investigate the effect of building plane shape parameters on total heating and cooling load. DesignBuilder was used to build and simulate individual building configuration. Energy consumption simulations for forty-eight U-shaped buildings with different plane layouts were performed to create a comprehensive dataset covering general ranges of side proportions of $U$-shaped buildings and building orientations. Statistical analysis was performed using MATLAB to develop a set of regression equations predicting energy consumption and optimizing floor shapes. Furthermore, perimeter-area ratio (PAR), width ratio, and depth ratio were considered as three factors to characterize the quantitative relationship between floor shape and energy consumption. It is envisioned that the binary quadratic polynomial regression models, visualized as a smooth surface in space and mapped to a vortex image on the plane, can be used to estimate the energy consumption in the early stages of the design when different building schemes and design concepts are being considered.
\end{abstract}

Keywords: building energy consumption; MATLAB; regression model; floor shape

\section{Research Background}

With the rapid development of China's urban construction and the continuous improvement of people's requirements for the built environment, the energy consumption of buildings continues to increase. In 2017, the total commercial energy consumption (standard coal) of building operations was 906 million tons, accounting for about $20 \%$ of the country's total energy consumption [1]. The energy consumption is predicted to continuously increase from the present, reaching a peak in the range between 1155 and 1243 million tons of standard coal equivalent in 2050 [2]. Based on meeting the requirements of the building environment, reducing the building operation energy consumption to achieve building energy efficiency has become one of the most important subjects for the sustainable development of buildings. A large amount of research at home and abroad has shown that the greatest potential for building energy efficiency comes from the design stage. The decisions made by architects during this stage will have a great impact on building performance in many aspects. For instance, by changing parameters of shape, orientation, and building envelope, the optimized scheme can save up to $40 \%$ of energy consumption [3,4] compared with the original scheme. As the design process advances, there is less and less room for building performance optimization, and the cost of getting the same benefits becomes higher and higher. 
In the early design stage, the building shape is one of the most important considerations, because it directly determines the building scale and the orientation of the building envelope. The building form can affect building performance in many aspects such as energy efficiency, construction cost, and aesthetic effects. Weimin Wang used genetic algorithms to study the influence of building floor shape on building performance in green building design and concluded that the variability of building shape can have various effects on building performance [5]. A study has shown that a mean annual energy consumption difference of $7.88 \%$ in favor of the prismatic building envelope comparing to buildings with right angles [6]. According to a study from MIT, four major factors are affecting building energy consumption: building design, climate, heating ventilation air conditioning systems (HVAC systems), and the personnel behavior model [7,8]. Personnel behavior patterns are very random. HVAC systems are improved by equipment engineers. The climate environment is fixed for each area. Therefore, building design plays an important role in effectively reducing building energy consumption.

The shape coefficient of the building has an important influence on building energy consumption and the incremental cost of building energy saving. In the energy saving evaluation, the shape coefficient is often considered as an evaluation index. Generally speaking, the lower the shape coefficient, the more beneficial it is to reduce building energy consumption, but this is only applicable in cold areas $[9,10]$. Even in cold areas, there is not an absolute proportional relationship between the shape coefficient and building energy consumption [11]. Specific shape of the building has a greater impact. In hot-summer and cold-winter areas, it faces two major issues in summer: cooling and dehumidification. It is difficult to find the balance point of shading and ventilation in design with just the shape coefficient. Research by Lin from Tongji University shows that the larger the shape coefficient of office buildings in hot-summer and cold-winter areas, the greater the potential for using natural resources and the lower the energy consumption of buildings [12]. It can be seen that the absolute value of the building shape coefficient is not significant for hot-summer and cold-winter regions. Wei analyzed the influence of the building's plane shape on the cooling load of the air-conditioning in the building, and pointed out that the building shape coefficient in the hot-summer and cold-winter area only needs to be controlled within a certain range [13]. Xia analyzed the performance of several buildings with different spatial layouts in Shanghai. It was concluded that no one shape can reduce heating energy consumption and cooling energy consumption at the same time when building shape changes [14]. In general, their research shows that the shape coefficient should not be used as a direct control factor in the generation of building forms. The influence mechanism of building forms on energy consumption is quite complicated.

Parameterization is an effective and cutting-edge method when considering the building performance in the design of the building form. Extending the concept of building shape coefficients into building shape parameters will be beneficial to further research on the relationship between building shape and energy consumption. A multivariate linear regression model was proposed to predict the energy consumption of office buildings under different standard floor plan shapes $[15,16]$. In this thesis, cold-dry climate and warm-humid climate were considered. Office buildings with seven shapes (rectangular, H-shaped, L-shaped, polygonal, triangular, T-shaped, and U-shaped) were simulated for the cooling and heating load. Research shows that the difference between regression prediction results and software simulation results is within $5 \%$. Some scholars also proposed a kind of design process building parametric modeling that combines evolutionary algorithms and energy consumption simulation to help architects better analyze building performance in the early scheme stage. This method first sets the variables and constraints among building design parameters, then performs modeling, design optimization, energy consumption and simulation. Whether the simulation results reach the expected value is a reference to decide on outputting the results or optimization and simulation again [17]. Li transformed the design issue into a mathematical model that was optimized through a genetic algorithm [18]. A new design workflow methodology was proposed, integrating evolutionary algorithms and energy simulation through Grasshopper for Rhinoceros 
3D [17]. It can be seen that with mathematical analysis and computer models, it is a reliable method to express the building form in the form of parameters to obtain the correlation between the building shape and energy consumption.

In the hot-summer and cold-winter area, U-shaped buildings are a widely distributed building layout. Their semi-enclosed plane form is not only conducive to ventilation and dehumidification but also relatively compact without excessive heat loss. The inner courtyard space is conducive to the construction of landscape gardens. Therefore, this paper takes U-shaped buildings as an example to study the correlation between the side proportion of building plane shape and building energy consumption. The parameter model of U-shaped building is constructed, with the help of MATLAB mathematical calculation software and DesignBuilder energy simulation software, and buildings with different layouts and their energy consumption data were obtained. Regression analysis of building shape parameters and energy consumption data was performed to predict the design parameters of low-energy U-shaped buildings.

\section{Methodology}

\subsection{Parametric Modeling of $U$-Shaped Buildings}

After investigating the existing buildings in hot-summer and cold-winter areas, it was found that the range of the orientation of U-shaped office buildings usually is south by west $40^{\circ}$ to south by east $40^{\circ}$. Entrances of buildings are mostly located on the notched side of the " $\mathrm{U}$ ". Therefore, SW $40^{\circ} \sim \mathrm{SE}$ $40^{\circ}$ is considered as a limiting condition for the orientation of building models. The field data are shown in Figure 1.

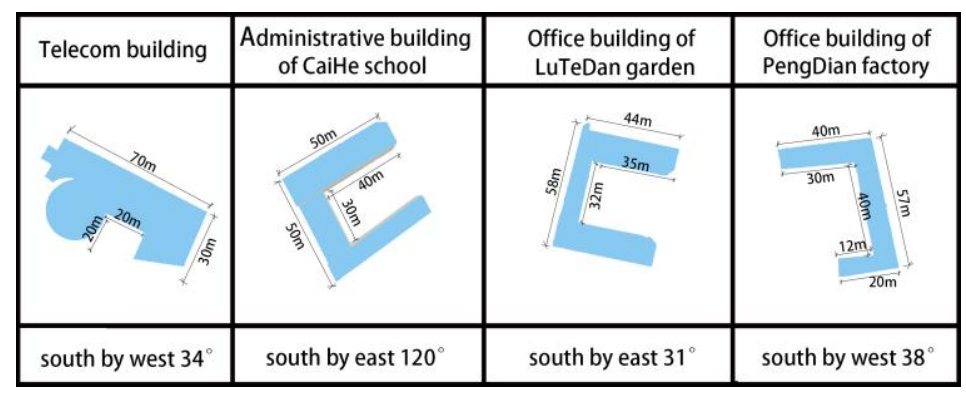

Figure 1. U-shaped office buildings in Hangzhou.

Retaining the main form characters of a U-shaped building, floor shape was simplified to construct the mathematical model. The southwest corner of the U-shaped building was taken as the coordinate origin and recorded as $\left(\mathrm{x}_{1}, \mathrm{y}_{1}\right)$. The remaining seven points were connected with line segments clockwise to generate a closed " $U$ ". The diagram is shown in Figure 2.

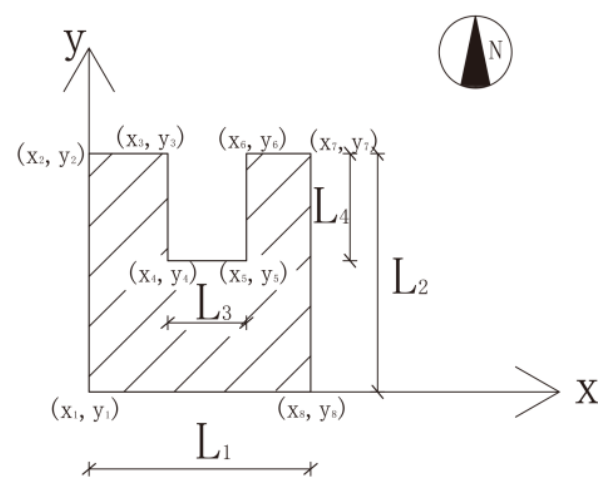

Figure 2. Parametric model of U-shaped buildings. 
In this paper, the building module was nine meters, which is a usual column-span value. MATLAB was used to generate corresponding building layouts by programming. Sixteen buildings layouts shown in Figure 3 were selected as characterized layouts among these generated models. South, south by east, and south by west were considered as building orientations to build models, as is shown in Figure 4. Parameters and data of building models are shown in Tables 1 and 2, respectively. These layout samples were selected by random-uniform sampling and subjective selection. Because the experimental model must conform to the practice of architecture design, after randomly generating data that meet the boundary conditions in MATLAB, the most likely solutions in the design were chose by the experience of architects.
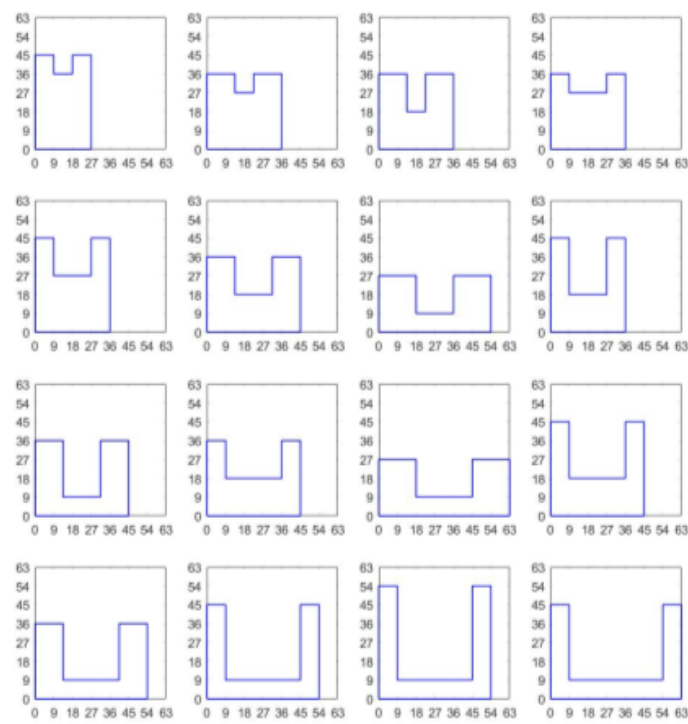

Figure 3. Building layouts generated by MATLAB.

\begin{tabular}{|c|c|c|c|c|c|c|c|}
\hline 1 & 2 & 3 & 4 & 5 & 6 & 7 & 8 \\
\hline ૫ & ப & 凹 & $\sqcup$ & ப & 凹 & س & 凹 \\
\hline$\beta$ & $\diamond$ & 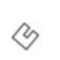 & $\diamond$ & $\Leftrightarrow$ & $\Leftrightarrow$ & ß & $\Leftrightarrow$ \\
\hline$\theta$ & $\theta$ & $\Leftrightarrow$ & $\otimes$ & $\Leftrightarrow$ & 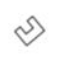 & $\Leftrightarrow$ & $\Leftrightarrow$ \\
\hline 9 & 10 & 11 & 12 & 13 & 14 & 15 & 16 \\
\hline 凹 & ப & كب & ษ & 凹 & $\amalg$ & 凹 & $\amalg$ \\
\hline 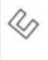 & $\Leftrightarrow$ & 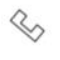 & $\nLeftarrow$ & $\nLeftarrow$ & $\mathbb{E}$ & $\mathbb{B}$ & $\mathbb{E}$ \\
\hline 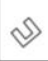 & $\curvearrowright$ & 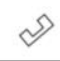 & 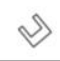 & $\triangleq$ & 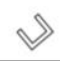 & $\gg$ & 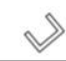 \\
\hline
\end{tabular}

Figure 4. Building layouts with different orientations.

Table 1. Parameters of building models.

\begin{tabular}{ccc}
\hline Constant Parameters & Design Parameters & Boundary Conditions \\
\hline Area: $12,000 \mathrm{~m}^{2}$ & Width $\mathrm{L}_{1}$ & $L 1 \times L 2-L 3 \times L 4=1200$ \\
Height: $36 \mathrm{~m}$ & Depth $\mathrm{L}_{2}$ & $\boldsymbol{L}_{1}>\boldsymbol{L}_{2}>\mathbf{1 2 0 0}$ \\
Floor: 10 & Notch width $\mathrm{L}_{3}$ & $\boldsymbol{L}_{1}>\boldsymbol{L}_{3}>\mathbf{0}$ \\
Floor area: $1200 \mathrm{~m}^{2}$ & Notch depth $\mathrm{L}_{4}$ & $\boldsymbol{L}_{2}>\boldsymbol{L}_{4}>\mathbf{0}$ \\
Floor height: $3.6 \mathrm{~m}$ & & \\
Window-wall ratio: 0.3 & & \\
\hline
\end{tabular}


Table 2. Data of building models.

\begin{tabular}{ccccccccc}
\hline No. & $\mathbf{L}_{\mathbf{1}} / \mathbf{m}$ & $\mathbf{L}_{\mathbf{2}} / \mathbf{m}$ & $\mathbf{L}_{\mathbf{3}} / \mathbf{m}$ & $\mathbf{L}_{\mathbf{4}} / \mathbf{m}$ & PAR & Width Ratio & Depth Ratio & Orientation \\
\hline 1 & 27 & 45 & 9 & 9 & 0.14 & 3.0 & 5.0 & SW, S, SE \\
2 & 36 & 36 & 9 & 9 & 1.14 & 4.0 & 4.0 & SW, S, SE \\
3 & 36 & 36 & 9 & 18 & 0.15 & 4.0 & 2.0 & SW, S, SE \\
4 & 36 & 36 & 18 & 9 & 0.14 & 2.0 & 4.0 & SW, S, SE \\
5 & 36 & 45 & 18 & 18 & 0.17 & 2.0 & 2.5 & SW, S, SE \\
6 & 45 & 36 & 18 & 18 & 0.17 & 2.5 & 2.0 & SW, S, SE \\
7 & 54 & 27 & 18 & 18 & 0.17 & 3.0 & 1.5 & SW, S, SE \\
8 & 36 & 45 & 18 & 27 & 0.18 & 2.0 & 1.7 & SW, S, SE \\
9 & 45 & 36 & 18 & 27 & 0.18 & 2.5 & 1.3 & SW, S, SE \\
10 & 45 & 36 & 27 & 18 & 0.17 & 1.7 & 2.0 & SW, S, SE \\
11 & 63 & 27 & 27 & 18 & 0.18 & 2.3 & 1.5 & SW, S, SE \\
12 & 45 & 45 & 27 & 27 & 0.20 & 1.7 & 1.7 & SW, S, SE \\
13 & 54 & 36 & 27 & 27 & 0.20 & 2.0 & 1.3 & SW, S, SE \\
14 & 54 & 45 & 36 & 36 & 0.23 & 1.5 & 1.3 & SW, S, SE \\
15 & 54 & 54 & 36 & 45 & 0.26 & 1.5 & 1.2 & SW, S, SE \\
16 & 63 & 45 & 45 & 36 & 0.24 & 1.4 & 1.3 & SW, S, SE \\
\hline
\end{tabular}

The coefficient of building shape (building external surface area/building volume) is an important factor affecting the heat consumption of a building. For the building plane, the shape coefficient formula can be transformed into "perimeter-area ratio" (PAR, building perimeter/building area). However, in hot-summer and cold winter regions, the shape coefficient is not suitable. Therefore, in this paper, it is proposed that building width ratio $\left(\mathrm{M}_{1}\right.$, width $\mathrm{L}_{1}$ /notch width $\left.\mathrm{L}_{3}\right)$ and building depth ratio $\left(\mathrm{M}_{2}\right.$, depth $\mathrm{L}_{2} /$ notch depth $\left.\mathrm{L}_{4}\right)$ be the performance index of the building plane to study the correlation between building plane form and energy consumption, as shown in Table 2 .

\subsection{Simulation of Building Energy Consumption}

DesignBuilder is a comprehensive user graphical interface simulation software developed for the building energy dynamic simulation program (Energy Plus). In this paper, building models were built in DesignBuilder v4.6.0, developed by DesignBuilder Software Ltd (London, UK), and the energy consumption calculation was performed using the Energy plus module in DesignBuilder. MATLAB got these simulation data and conducted regression analysis.

The office building was the building type studied in this paper. All buildings were simulated in DesignBuilder, as shown in Figure 5. The schedule used in this simulation was "Office_OpenOff_Occ" in DesignBuilder. The most common workday schedule in office is shown in Figure 6. The weather file was derived from ASHRAE (American Society of Heating, Refrigerating and Air-Conditioning Engineers, Inc.), and the location in simulation is Hangzhou, China. In this area, winter lasts from December to February, summer lasts from June to August. The annual sunshine duration reaches $1879.8 \mathrm{~h}$, and the annual sunshine percentage is $42 \%$. SSW (south-southwest) is the prevailing wind direction in summer, with an average wind speed of $2.2 \mathrm{~m} / \mathrm{s}$. NNW (north-northwest) is the prevailing wind direction in winter, with an average wind speed of $2.3 \mathrm{~m} / \mathrm{s}$. Temperature of the hottest month is $28.5^{\circ} \mathrm{C}$, and the coldest month temperature is $3.7^{\circ} \mathrm{C}$. Relative humidity is $77-80 \%$ throughout the year. In the modeling, building materials were "Best practice, Medium weight" derived from "early design template" in DesignBuilder. The outer walls were made up of four layers: brickwork $100 \mathrm{~mm}$, XPS (extruded polystyrene) $100 \mathrm{~mm}$, concrete block $100 \mathrm{~mm}$, and gypsum plastering $10 \mathrm{~mm}$. HVAC systems used "Fan coil Unit (4-pipe), Air cooled Chiller". Both natural ventilation and mechanical ventilation were considered in this paper. The shading system took into account window shading. Because this article is a comparative study of building energy consumption of different building forms, it is necessary to ensure that all simulations adopt exactly the same settings. 


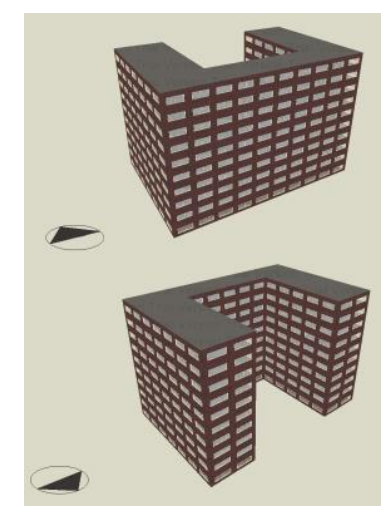

(a) Building model

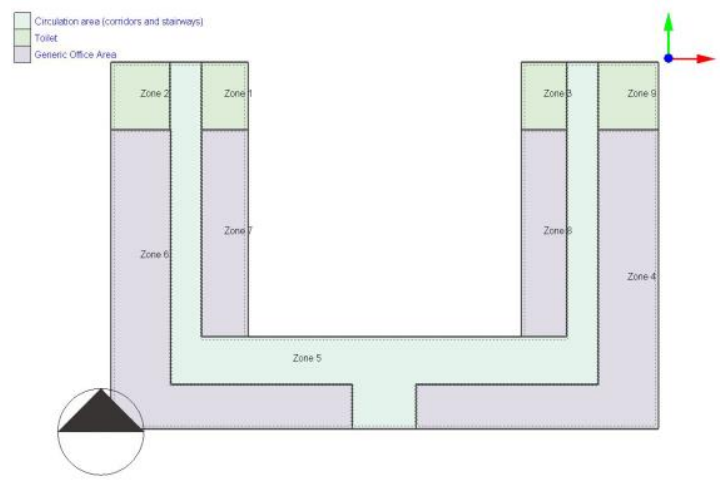

(b) Building space division

Figure 5. Building models and space division in DesignBuilder.

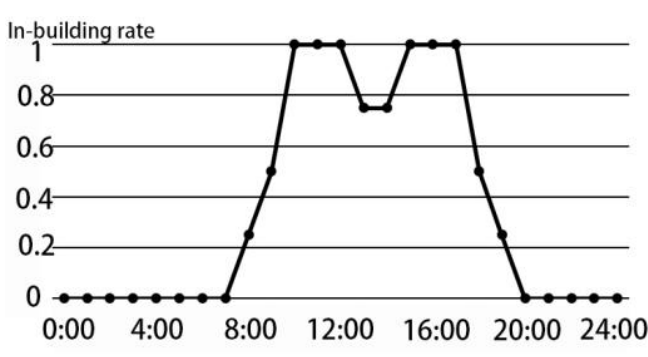

(a) In-building rate

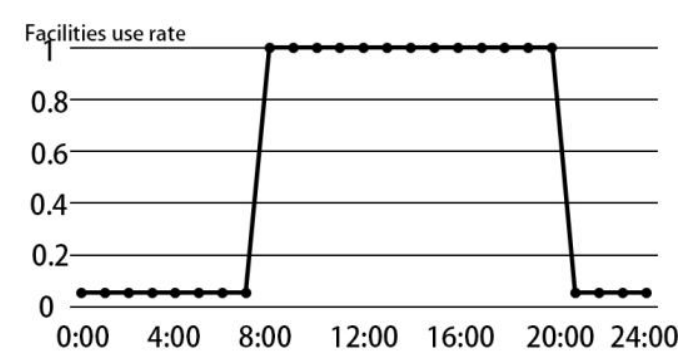

(b) Facilities use rate

Figure 6. Workday schedule in the office.

Table 3 shows the model data of simulation. The results of energy consumption simulation are presented in Table 4.

Table 3. Model data of simulation.

\begin{tabular}{cc}
\hline Activity & Data \\
\hline Occupancy & 0.1110 people $/ \mathrm{m}^{2}$ \\
Target illuminance & $400 \mathrm{lux}$ \\
Cooling setpoint temperature & $24^{\circ}$ \\
Cooling set back & $28^{\circ}$ \\
Heating setpoint temperature & $22^{\circ}$ \\
Heating set back & $12^{\circ}$ \\
Office equipment & $11.77 \mathrm{w} / \mathrm{m}^{2}$ \\
Domestic hot water & $0.2 \mathrm{~L} / \mathrm{m}^{2}$-day \\
\hline
\end{tabular}

There is a large deviation in model SE9, which should be removed as an outlier when doing data analysis. It can be seen from Figure 7 that under common orientations (SW $40^{\circ} \sim \mathrm{SE} 40^{\circ}$ ), there is no significant correlation between building energy consumption and orientation azimuth. The energy consumption corresponding to the layout of each building model is as follows: layouts $1-7<$ layouts $8-13<$ layouts $14-16$, rising in steps. The quantitative equation of this step-like relationship was studied by the mathematical analysis below. 
Table 4. Total energy consumption.

\begin{tabular}{|c|c|c|c|c|c|}
\hline & Orientation & $\begin{array}{c}\text { Energy Consumption } \\
\left(\mathrm{kWh} / \mathrm{m}^{2} \cdot \mathbf{a}\right)\end{array}$ & No. & Orientation & $\begin{array}{c}\text { Energy Consumption } \\
\left(\mathrm{kWh} / \mathrm{m}^{2} \cdot \mathbf{a}\right)\end{array}$ \\
\hline & $S$ & 91 & & $S$ & 95 \\
\hline \multirow[t]{3}{*}{1} & SW & 91 & 9 & SW & 95 \\
\hline & SE & 91 & & SE & 115 \\
\hline & $\mathrm{S}$ & 89 & & $\mathrm{~S}$ & 94 \\
\hline \multirow[t]{3}{*}{2} & SW & 90 & 10 & SW & 94 \\
\hline & SE & 90 & & SE & 94 \\
\hline & $S$ & 91 & & $\mathrm{~S}$ & 94 \\
\hline \multirow[t]{3}{*}{3} & SW & 92 & 11 & SW & 94 \\
\hline & $\mathrm{SE}$ & 92 & & SE & 94 \\
\hline & $S$ & 90 & & $S$ & 94 \\
\hline \multirow[t]{3}{*}{4} & SW & 91 & 12 & SW & 94 \\
\hline & SE & 91 & & SE & 94 \\
\hline & $S$ & 92 & & $S$ & 95 \\
\hline \multirow[t]{3}{*}{5} & SW & 92 & 13 & SW & 95 \\
\hline & SE & 92 & & SE & 95 \\
\hline & S & 91 & & $\mathrm{~S}$ & 100 \\
\hline \multirow[t]{3}{*}{6} & SW & 92 & 14 & SW & 100 \\
\hline & SE & 92 & & SE & 100 \\
\hline & $S$ & 92 & & $S$ & 100 \\
\hline \multirow[t]{3}{*}{7} & SW & 94 & 15 & SW & 100 \\
\hline & SE & 94 & & SE & 100 \\
\hline & $S$ & 95 & & $S$ & 100 \\
\hline \multirow[t]{2}{*}{8} & SW & 95 & 16 & SW & 100 \\
\hline & SE & 95 & & SE & 100 \\
\hline
\end{tabular}

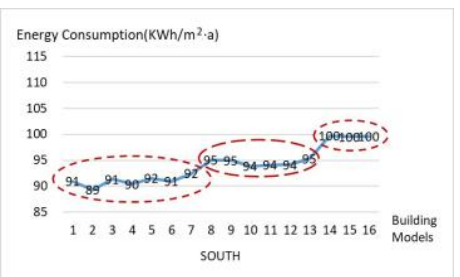

(a) South buildings

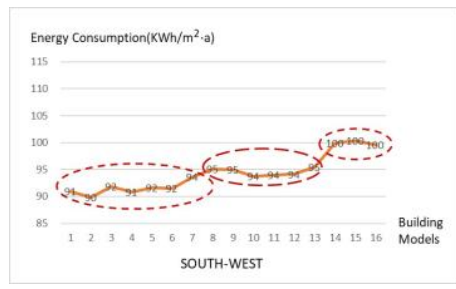

(b) Southwest buildings

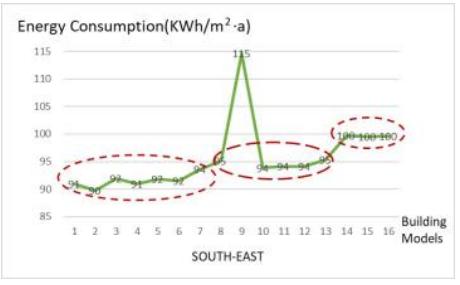

(c) Southeast buildings

Figure 7. Building "plane layouts—energy consumption" in different orientations.

\section{Results and Discussion}

In this study, perimeter-area ratio (PAR), width ratio $\left(\mathrm{M}_{1}\right)$, and depth ratio $\left(\mathrm{M}_{2}\right)$ of the building models were selected as the design parameters for studying the relationship between building shape and building energy consumption. A univariate linear regression model of energy consumption on PAR, a univariate nonlinear regression model of energy consumption on width ratio/depth ratio, and a binary polynomial regression model of energy consumption on width ratio and depth ratio were constructed. Through regression analysis and variance analysis, it was found that the binary quadratic equation can well fit the quantitative relationship between building energy consumption and width ratio and depth ratio. The fitting results were better than that of the linear model of energy consumption on perimeter-area ratio.

\subsection{Perimeter-Area Ratio and Energy Conusmption of Buildings}

Perimeter-area ratio is a variant of building shape coefficient. In this paper, the effect of perimeter-area ratio on energy consumption of buildings with different orientations was analyzed, by establishing a univariate linear regression model of building energy consumption and perimeter-area 
ratio of building standard floor plan (Tables 5-7). It was found that perimeter-area ratio had the greatest influence on buildings facing south. The slope of the first-order function of southward buildings whose energy consumption changed with PAR was 93.46, while the slopes of buildings facing southwest and southeast were 87.58 and 85.33 , respectively. Therefore, in the perspective of perimeter-area ratio, the influence degree of building energy consumption on building orientation in hot summer and cold winter regions is southward > southwestward > southeastward. That is, for buildings facing south, the design should pay more attention to the influence of the plane form on energy consumption.

Table 5. "Perimeter-area ratio and energy consumption" of southward buildings.

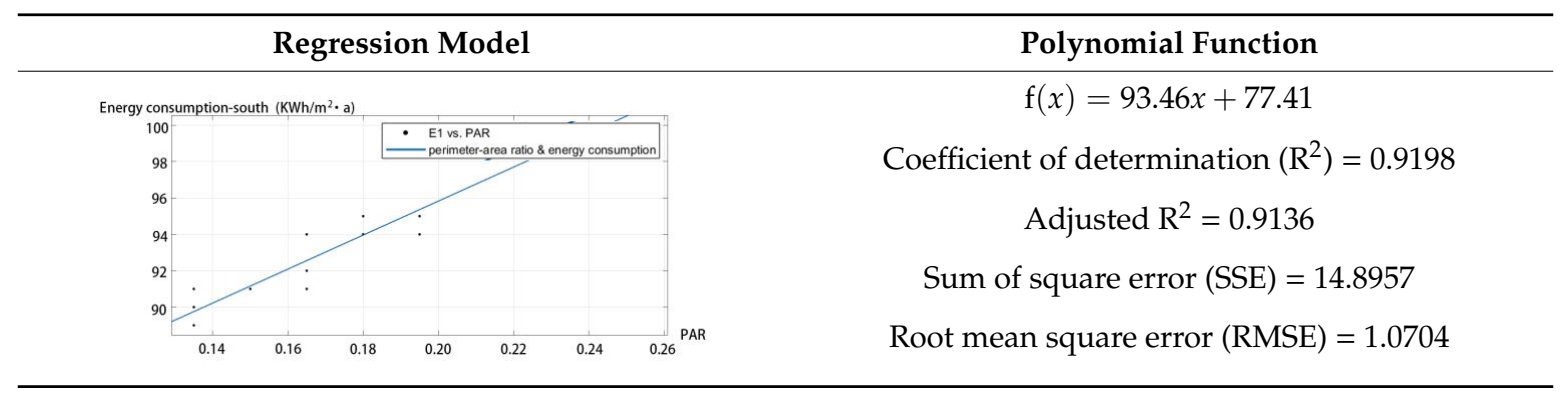

Table 6. "Perimeter-area ratio and energy consumption" of southwestward buildings.

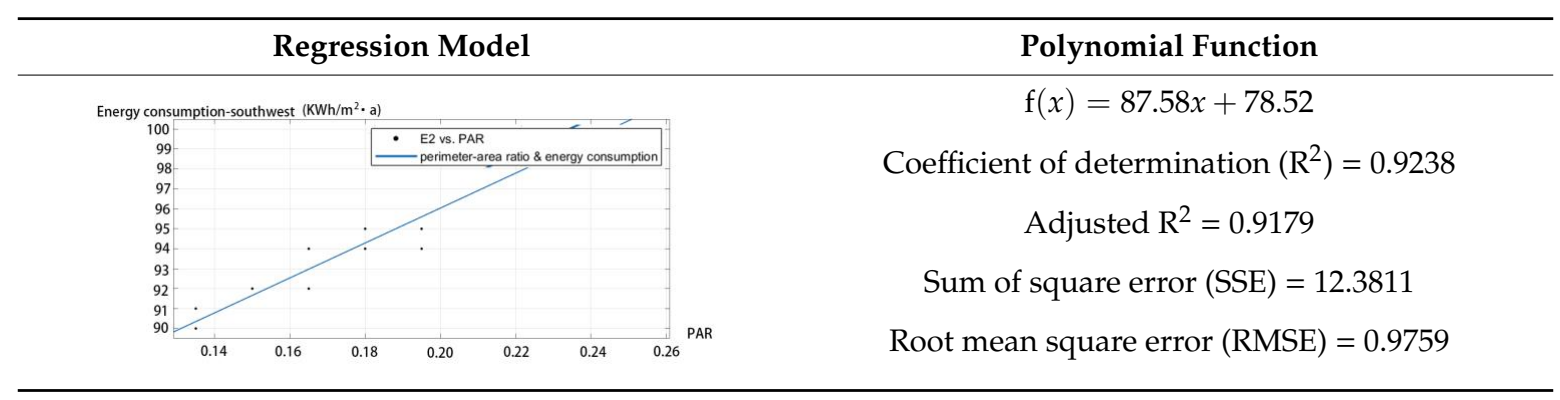

Table 7. "Perimeter-area ratio and energy consumption" of southeastward buildings.

\begin{tabular}{|c|c|}
\hline Regression Model & Polynomial Function \\
\hline Energy consumption-southeast $\left(\mathrm{KWh} / \mathrm{m}^{2} \cdot\right.$ a) & $\mathrm{f}(x)=85.33 x+78.99$ \\
\hline - E3v. PAR & Coefficient of determination $\left(R^{2}\right)=0.9192$ \\
\hline $\begin{array}{l}97 \\
96 \\
95\end{array}$ & Adjusted $\mathrm{R}^{2}=0.9130$ \\
\hline $\begin{array}{l}94 \\
93 \\
92\end{array}$ & Sum of square error $(\mathrm{SSE})=12.5222$ \\
\hline $0.22 \quad 0.24 \quad 0.26$ PAR & Root mean square error $($ RMSE $)=0.9815$ \\
\hline
\end{tabular}

\subsection{Width Ratio, Depth Ratio, and Energy Consumption of Buildings}

A U-shaped building's plane form can be characterized by width ratio and depth ratio. Firstly, a regression analysis of the correlation between single factor and energy consumption was conducted. Then, the regression model considering simultaneously the effect of width ratio and depth ratio on energy consumption was established. It was indicated that the double-factor effect model is better than the single characterization model, and better than the model of PAR. In residual plots of Tables $8-16$, it can be seen that the residuals were random, but there were several observation data points that deviate greatly from the established model, so they should be eliminated as outliers. After removing the abnormal points, the points of the residual graph were all in $(-2,2)$, and there was no trend. 
Table 8. "Width ratio and energy consumption" of southward buildings.

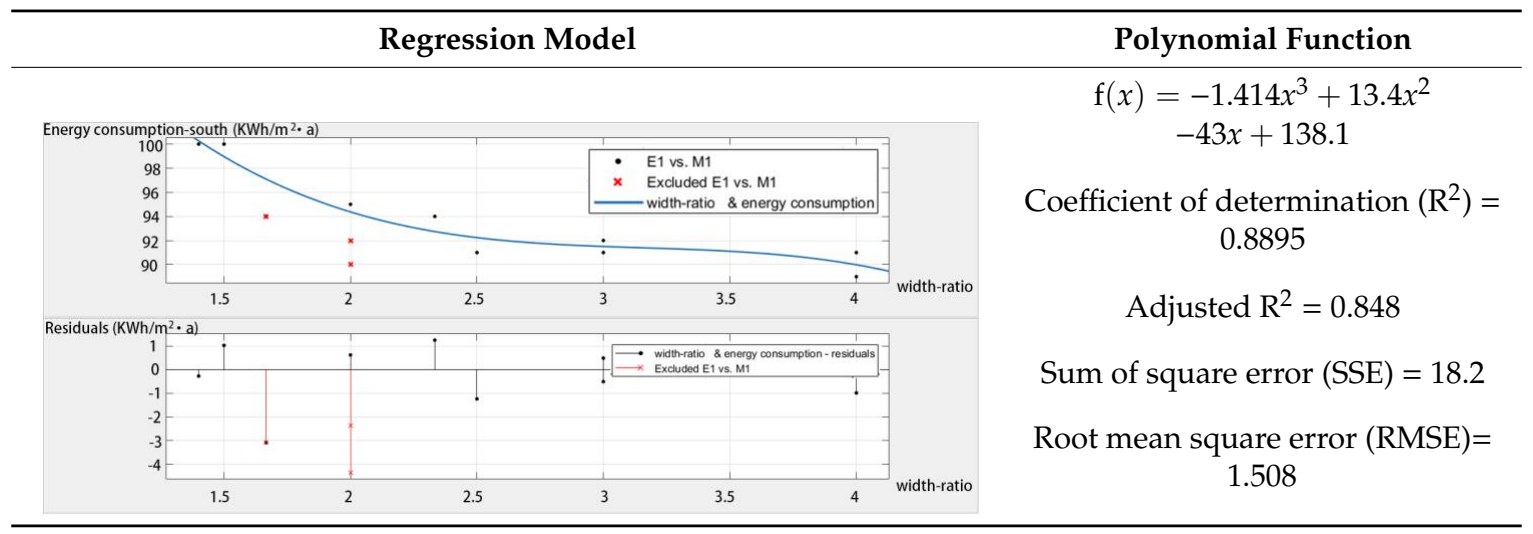

Table 9. "Width ratio and energy consumption" of southwestward buildings.

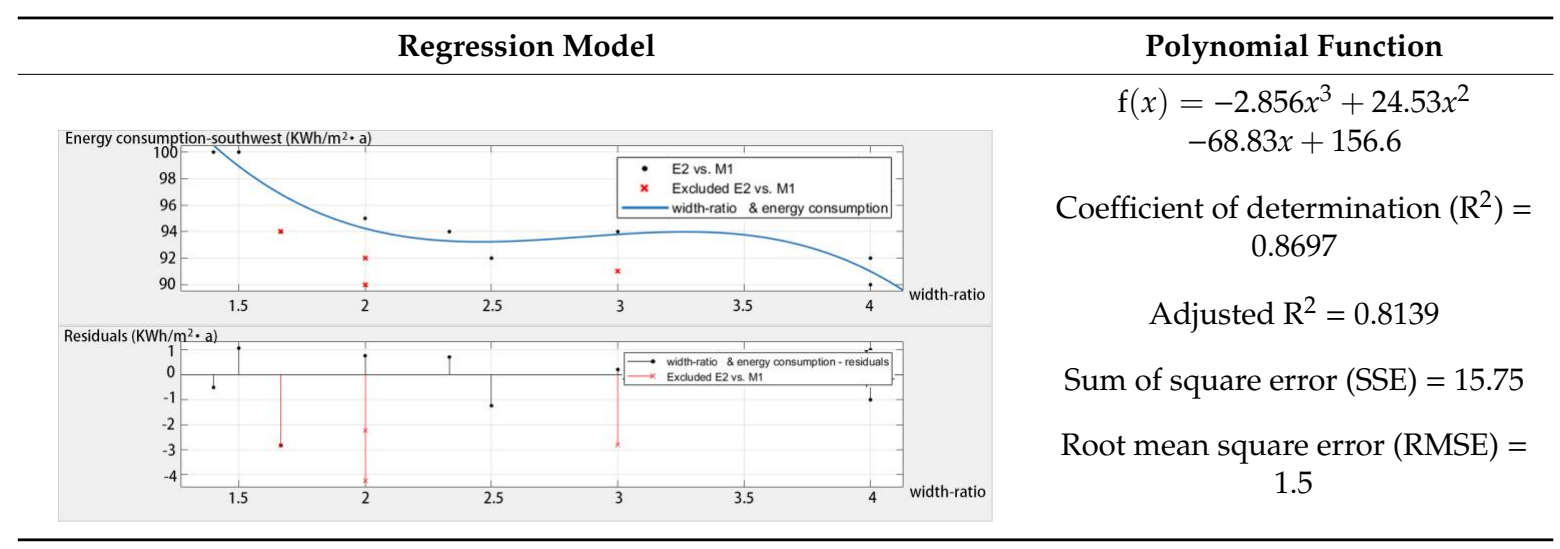

Table 10. "Width ratio and energy consumption" of southeastward buildings.

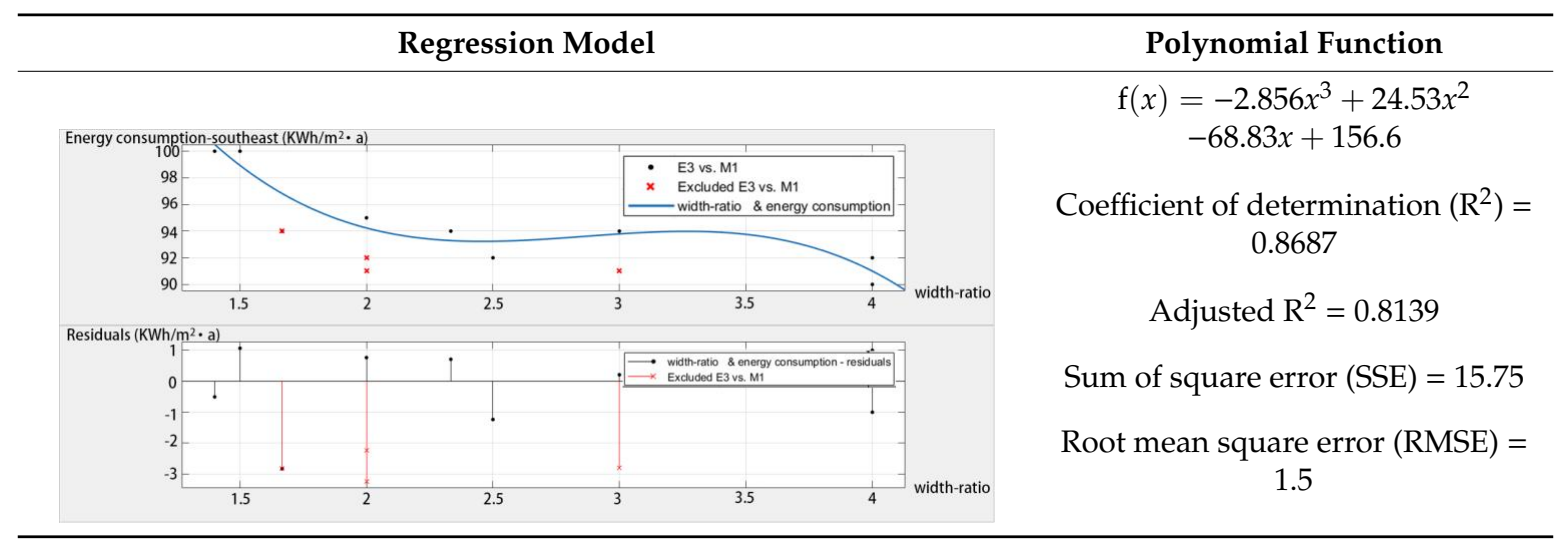


Table 11. "Depth ratio and energy consumption" of southward buildings.

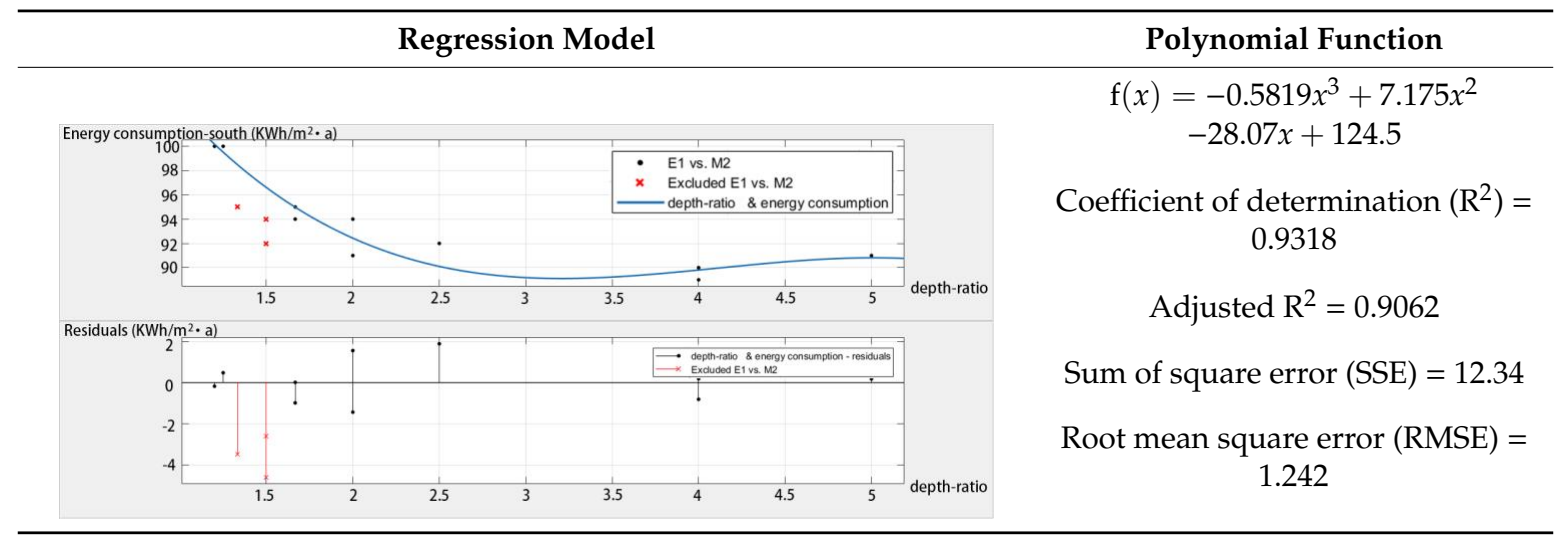

Table 12. "Depth ratio and energy consumption" of southwestward buildings.

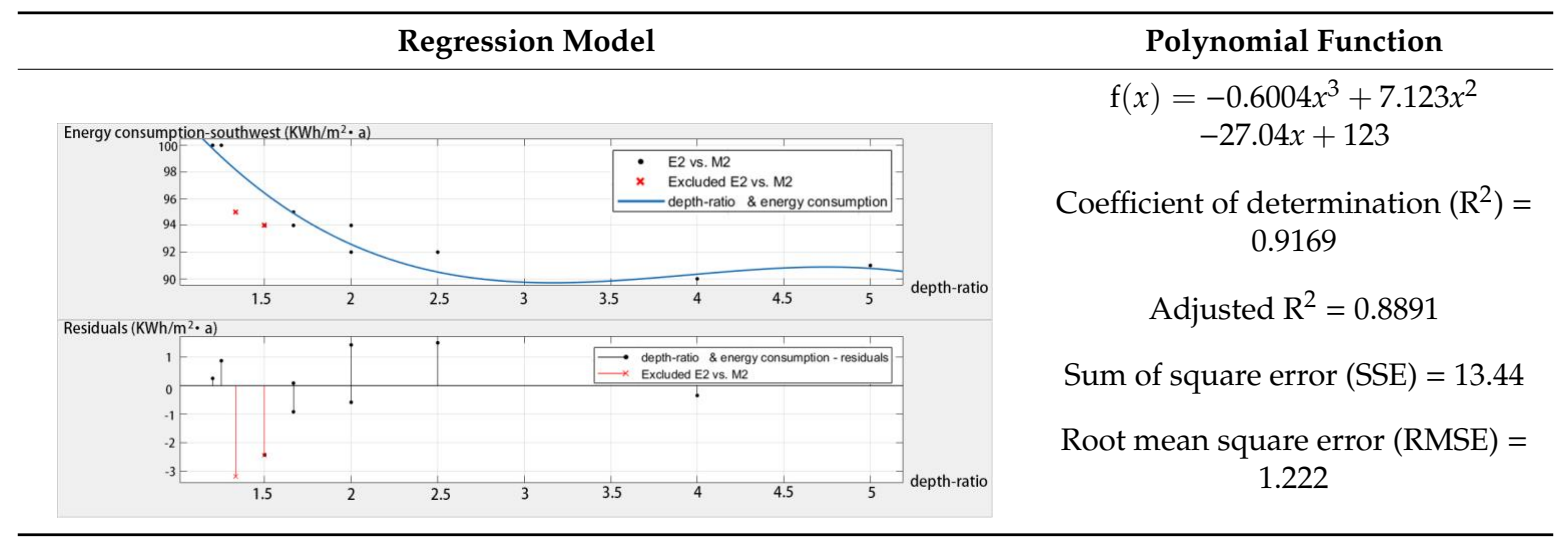

Table 13. "Depth ratio and energy consumption" of southeastward buildings.

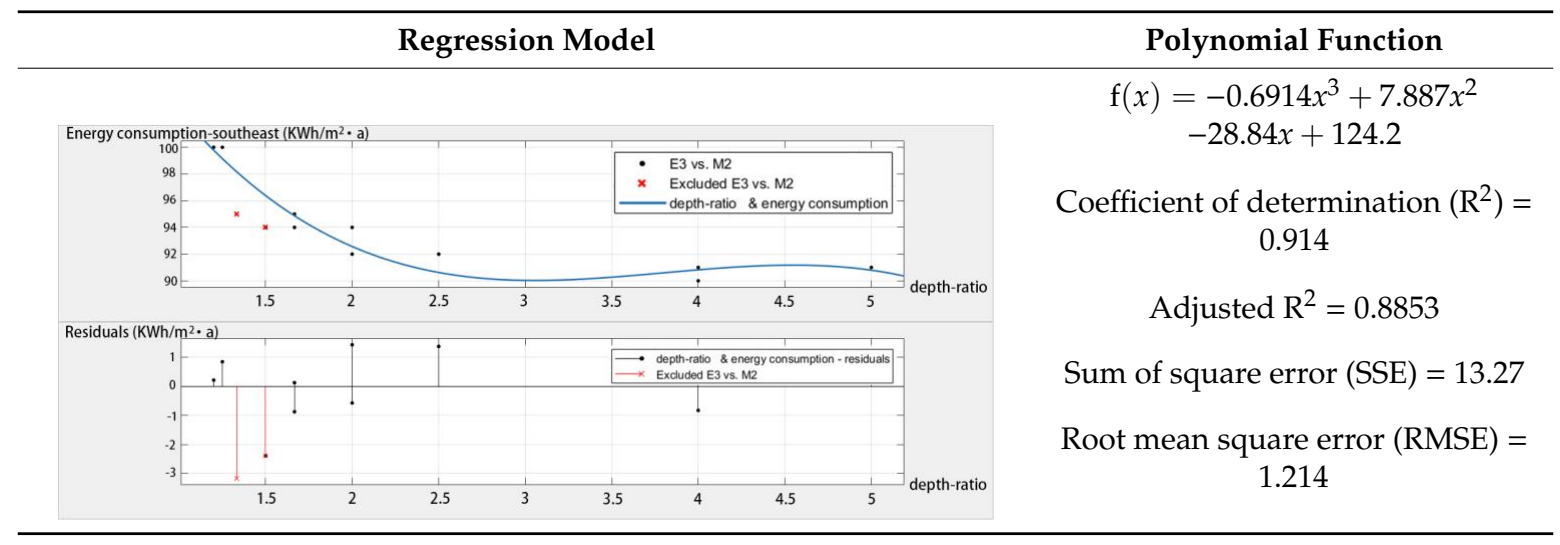


Table 14. "Width ratio, depth ratio, and energy consumption" of southward buildings.

\begin{tabular}{ccccccc}
\hline \multicolumn{10}{c}{ Regression Model } & $\begin{array}{c}\text { Polynomial Function } \\
\mathrm{f}(x, y)=91-2.8 x-4 y \\
+1.33 x^{2}+0.34 x y+2 y^{2}\end{array}$ \\
\hline \\
\hline
\end{tabular}

Table 15. "Width ratio, depth ratio, and energy consumption" of southwestward buildings.

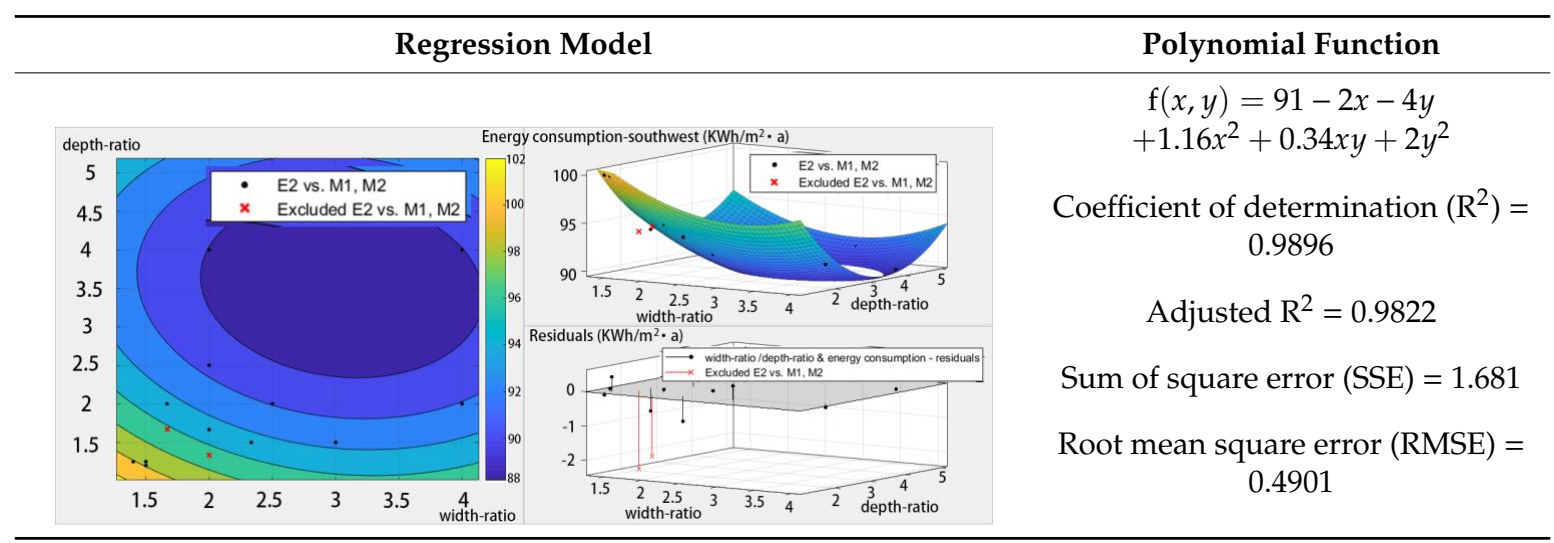

Table 16. "Width ratio, depth ratio, and energy consumption" of southeastward buildings.

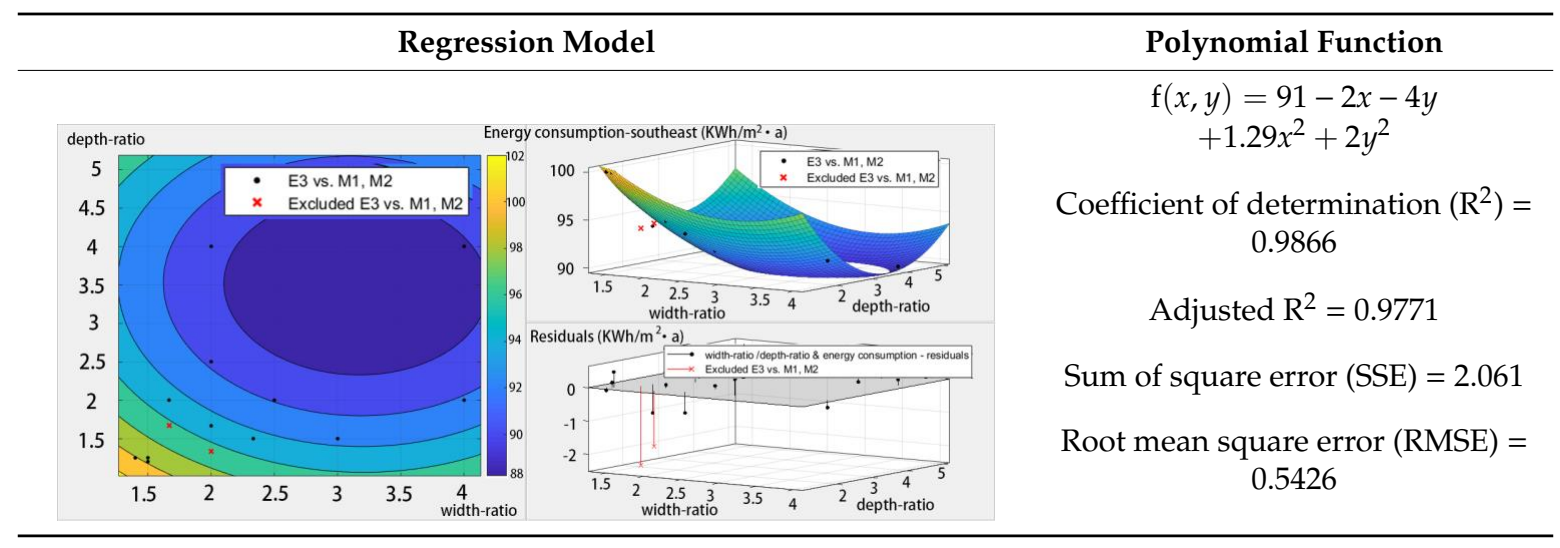

\subsubsection{Width Ratio and Energy Consumption of Buildings}

Above 95\% confidence level, regression models were constructed for width ratio and energy consumption of buildings with different orientations (Tables 8-10). It was suggested that the cubic function model can characterize regression models with a coefficient of determination above 0.8 . Building energy consumption generally decreases as width ratio increases, that is, the narrower the notch of the " $U$ ", the lower the energy consumption level. When the width ratio was between 1.5 and 2 , energy consumption decreased greatly as width ratio increased; when the width ratio was between 2 and 4, building energy consumption slowly decreased as width ratio increased.

For southward buildings, there were two parts in the function curves as shown in Table 8 . When the width ratio was between 1.5 and 2.5, the slope of changes of energy consumption with the width ratio 
reached $9 \mathrm{kWh} / \mathrm{m}^{3} \cdot \mathrm{a}$. That is, when the width ratio was reduced by one meter, electricity consumption per square meter would be reduced by $9 \mathrm{kWh}$ per year. When the width ratio was between 2.5 and 4 , the slope of changes of energy consumption with width ratio was $0.67 \mathrm{kWh} / \mathrm{m}^{3} \cdot \mathrm{a}$. That is, for every meter reduction of width ratio in $\mathrm{U}$-shaped buildings, $0.67 \mathrm{kWh}$ electricity consumption per square meter of a building could be reduced each year.

For southwestward buildings, there were three parts in the function curves as shown in Table 9. When the width ratio was between 1.5 and 2, the slope of changes of energy consumption with width ratio reached $12 \mathrm{kWh} / \mathrm{m}^{3} \cdot \mathrm{a}$. That is, when width ratio was reduced by one meter, electricity consumption per square meter would be reduced by $12 \mathrm{kWh}$ per year. When the width ratio was between 2 and 3.5, energy consumption varied little with width ratio and remained unchanged. When the width ratio was between 3.5 and 4, the slope of changes of energy consumption with width ratio was $6 \mathrm{kWh} / \mathrm{m}^{3} \cdot \mathrm{a}$, which means that for every meter reduction in width ratio, $6 \mathrm{kWh}$ electricity consumption per square meter of a building could be reduced each year. The trend of building energy consumption on width ratio for southeastward buildings is consistent with that of southwestward buildings, as shown in Table 10.

Briefly, under the common orientation (SW $\left.40^{\circ} \sim \mathrm{SE} 40^{\circ}\right)$, the correlation between width ratio and energy consumption of southward buildings is more stable. Therefore, the fitting result is more reliable. In contrast, for southwest-facing buildings and southeast-facing buildings, regression models of energy consumption on width ratio require more cases to illustrate.

\subsubsection{Depth Ratio and Energy Consumption of Buildings}

Same as above, with a confidence level of more than 95\%, regression models for depth ratio and energy consumption of buildings with different orientations were constructed (Tables 11-13). Studies have shown that cubic function polynomials can characterize regression models with decision coefficients above 0.9. According to these function images, the curve was divided into two sections. Energy consumption of one part decreased significantly with depth ratio increased, and the other one was unchanged, the slope of the descending section reached $10 \sim 16 \mathrm{kWh} / \mathrm{m}^{3} \cdot \mathrm{a}$. It followed that the effect of depth ratio on energy consumption was significantly greater than the effect of width ratio on energy consumption by comparing with Tables $8-10$.

Moreover, the function images in Tables 11-13 were the same, indicating that the relationship between building depth ratio and energy consumption was hardly affected by orientation, which was relatively stable in general building orientations (SW $40^{\circ} \sim \mathrm{SE} 40^{\circ}$ ).

\subsubsection{Width Ratio, Depth Ratio, and Energy Consumption of Buildings}

Considering the influence of width ratio and depth ratio on building energy consumption, it is necessary to establish a double-factor effect model. Through data fitting, a binary quadratic function with a 95\% confidence level was found as the regression model, as is shown in Tables 14-16. In these figures, different colors indicated different energy consumption values. The darker the color, the lower the energy consumption, and vice versa. The model image was presented as a smooth surface in space, which is mapped into a vortex on the plane, which showed that there was an optimal area with low energy consumption (dark vortex kernel in the picture) for the plane form of buildings. A low-energy building shape will be created with the width ratio and depth ratio in this area. Moreover, those images explained the phenomenon that energy consumption stepwise raised with layout changes in Figure 7: Layouts 1-7 were in the dark area of the vortex center, layouts 8-13 were in the light-colored area of the vortex center, and layouts 14-16 were in the bright areas of vortex at the edges, so their energy consumption was: layouts 1-7 < layouts 8-13 < layouts 14-16.

For southward buildings, the determination coefficient of the regression model reached 0.99, which was 0.94 and 0.98 for southwestern buildings and southeastward buildings, suggesting that the fitting effect of southward building models was better. Furthermore, the low-energy core area of 
south-facing buildings was smaller than that of the other two orientations, which indicated that the floor shape had a greater impact on energy consumption when facing south.

\section{Conclusions}

In this paper, the coupling relationship between floor shape and energy consumption of U-shaped office buildings in hot-summer and cold-winter areas was studied, comparing the influence of perimeter-area ratio, width ratio, and depth ratio of the standard floor on energy consumption. A ladder-like relationship and swirling images between layouts and energy consumption were found. The three kinds of regression models were compared in the article not only validated fitting results but also improved the determination coefficient of regression models to 0.99 . The binary fitting regression model of energy consumption to width ratio and depth ratio was better than energy consumption to perimeter-area ratio, better than the single-factor fitted regression model of energy consumption to width ratio or depth ratio $\left(R^{2}: 0.99>0.9>0.8\right)$. Based on field survey data, combined programming language, the paper built a parametric model of $U$-shaped buildings to analyze the influence of building shape on energy performance by regression analysis of building parameters and energy consumption data through data fitting. This research methodology on the relationship between building shape and performance is theoretically applicable to optimization analysis of building form to performance in various climatic regions. The hot summer and cold winter area in China is the most uncomfortable zone in the same latitude area. Architects tend to be unsure of how to optimize building forms based on energy performance in the first stage of design. Some research suggested that the coefficient of building shape in this area need only be controlled within a certain range [12]. This paper has carried out a quantitative study on this "suitable range" and provide a basis for comparison and selection of U-shaped buildings.

Main conclusions:

1. From the perspective of building energy consumption, perimeter-area ratio has a greater impact on buildings facing south than on other building orientations.

2. For U-shaped buildings, the influence of depth ratio on energy consumption is greater than that of width ratio.

3. The quantified relationship between building energy consumption and width ratio and depth ratio can be represented by a binary quadratic function, which is visualized as a smooth surface in space, mapped onto a plane as a vortex image. The more the value of width ratio and depth ratio is off-center, the higher the energy consumption.

4. The quantitative relationships between width ratio, depth ratio, and energy consumption of U-shaped buildings in hot-summer and cold-winter areas are shown in Table 17.

5. Architects can intuitively select the appropriate width ratio and depth ratio from "floor shape-energy consumption" map for design or estimate energy consumption based on the mapping picture to optimize design scheme, as shown in Figure 8.

Table 17. The quantitative relationships between width ratio, depth ratio, and energy consumption.

\begin{tabular}{cc}
\hline Building Orientations & Function \\
\hline Southward buildings & $\mathrm{f}(x, y)=91-2.8 x-4 y+1.33 x^{2}+0.34 x y+2 y^{2}$ \\
Southwestward buildings & $\mathrm{f}(x, y)=91-2 x-4 y+1.16 x^{2}+0.34 x y+2 y^{2}$ \\
Southeastward buildings & $\mathrm{f}(x, y)=91-2 x-4 y+1.29 x^{2}+2 y^{2}$ \\
\hline
\end{tabular}

Note: $x$ represents width ratio, $y$ represents depth ratio, and $f(x, y)$ represents building energy consumption value. 


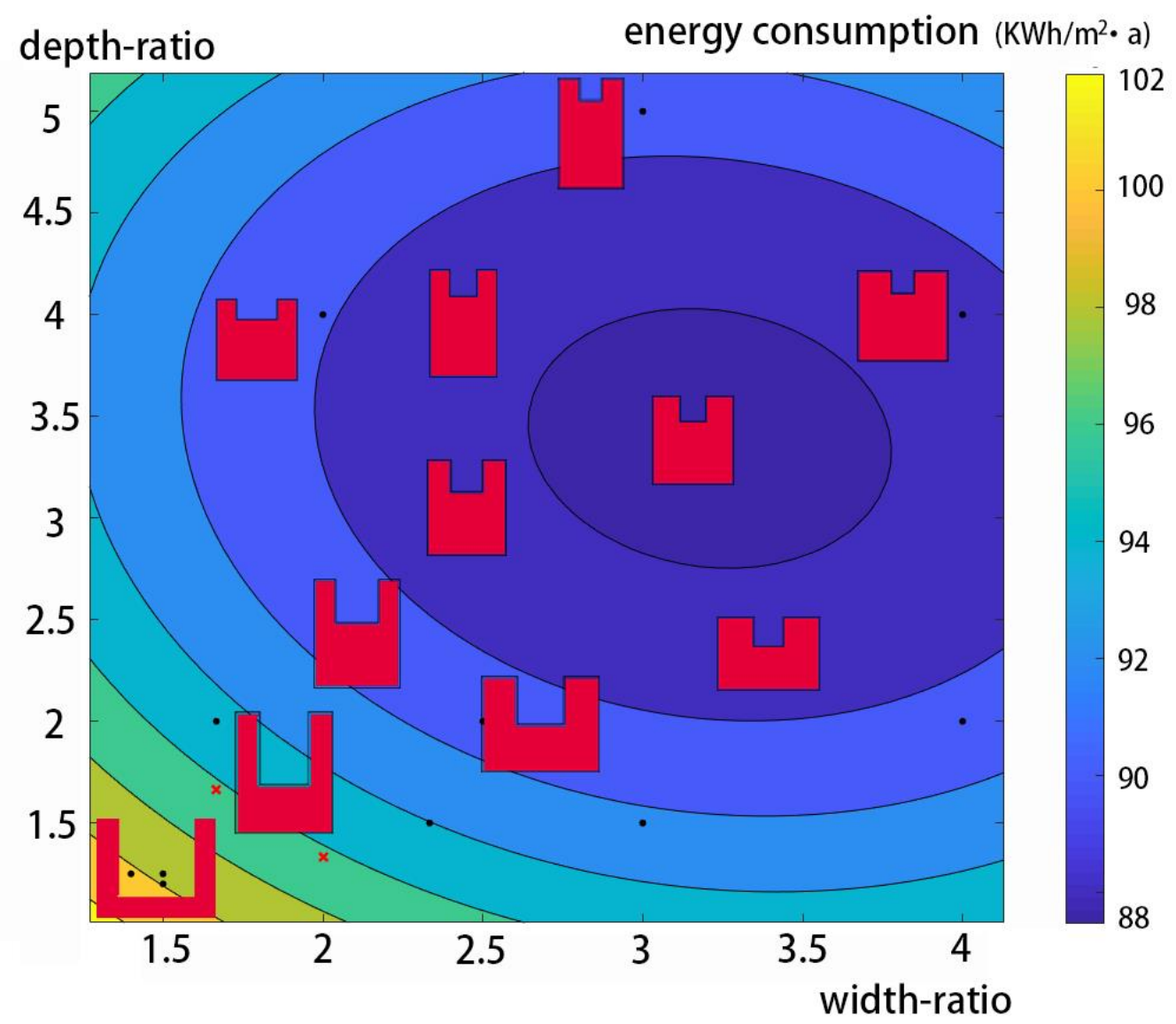

Figure 8. The mapping of "floor shape-energy consumption" for southward U-shaped buildings.

Author Contributions: Methodology, X.Y.; software, W.L.; validation, X.Y.; formal analysis, W.L.; investigation, W.L.; resources, X.Y.; writing-original draft preparation, W.L.; writing—review and editing, X.Y.; supervision, $X . Y$;; funding acquisition, X.Y. All authors have read and agreed to the published version of the manuscript.

Funding: This research was funded by the National Natural Science Foundation of China, grant number 51878608; the Natural Science Foundation of Zhejiang Province, grant number LY18E080025; and the Self-declared Social development Foundation of Hangzhou, grant number 20180533B08.

Conflicts of Interest: The authors declare no conflict of interest. The funders had no role in the design of the study; in the collection, analyses, or interpretation of data; in the writing of the manuscript; or in the decision to publish the results.

\section{References}

1. Building Energy Research Center, Tsinghua University. Annual Research Report on Building Energy Conservation; Building Energy Research Center, Tsinghua University: Beijing, China, 2018.

2. Guangyue, X.; Weiming, W. China's energy consumption in construction and building sectors: An outlook to 2100. Energy 2020, 195, 1-12.

3. Nick, B.; Koen, S. Energy and Environment in Architecture: A Technical Design Guide; E\&FN Spon: New York, NY, USA, 2000.

4. Brophy, V.; Lewis, J.O. A Green Vitruvius-Principles and Practice of Sustainable Architecture Design, 2nd ed.; Routledge: London, UK, 2011.

5. Weiming, W.; Hugues, R.; Radu, Z. Floor shape optimization for green building design. Adv. Eng. Inform. 2006, 363-378. [CrossRef]

6. Stelios, C.Z.; Christos, A.T. The role of building form in energy consumption: The case of a prismatic building in Athens. Energy Build. 2012, 48, 97-102. 
7. Carlo, R.; Nick, B.; Koen, S. Energy consumption and urban texture. Energy Build. 2005, 37, 762-776.

8. Koen, S. Energy and the city: Density, buildings and transport. Energy Build. 2003, 35, 3-14.

9. Hongyuan, M.; Fei, W.; Yuliang, Z. Study on the design for architecture form adapted to cold regions for low energy consumption. Archit. J. 2013, 11, 88-93.

10. Shan, H.; Da, Y.; Ying, C. Influence of building space form on the energy consumption of residential buildings. Build. Sci. 2015, 31, 117-124.

11. Binbin, R.; Yiping, W.; Shaoying, X. Forms of office buildings with low energy consumption in Tianjin area. Build. Energy Effic. 2015, 43, 66-68.

12. Meishun, L.; Yiqun, P.; Huaiding, L. Influence of building body-type coefficient on energy consumption of office buildings in hot-summer-and cold-winter areas of China. Build. Energy Effic. 2015, 10, 63-66.

13. Huang, W.; Linfang, Z.; He, J.; Houli, S. The analysis of the building's plane shape influence on its energy consumption. In Proceedings of the International Conference on E-Product E-Service and E-Entertainment, Henan, China, 7-9 November 2010.

14. Bing, X. Low carbon design research on the space layout types of office building. New Archit. 2016, 11, 92-95.

15. Mottahedi, M.; Mohammadpour, A.; Shams Amiri, S.; Riley, D.; Asadi, S. Multi-Linear regression models to predict the annual energy consumption of an office building with different shapes. Procedia Eng. 2015, 118, 622-629. [CrossRef]

16. Asadi, S.; Shams Amiri, S.; Mottahedi, M. On the development of multi-linear regression analysis to assess energy consumption in the early stages of building design. Energy Build. 2014, 85, 246-255. [CrossRef]

17. Touloupaki, E.; Theodosiou, T. Optimization of building form to minimize energy consumption through parametric modelling. Procedia Environ. Sci. 2017, 38, 509-514. [CrossRef]

18. Li, L. The optimization of architectural shape based on genetic algorithm. Front. Archit. Res. 2012, 1, 392-399. [CrossRef]

(C) 2020 by the authors. Licensee MDPI, Basel, Switzerland. This article is an open access article distributed under the terms and conditions of the Creative Commons Attribution (CC BY) license (http://creativecommons.org/licenses/by/4.0/). 